\title{
Verification of NADH content measurements by portable optical diagnostic system in living brain tissue
}

E. Zherebtsov, P. Angelova, S. Sokolovski, A. Abramov, E. Rafailov

E. Zherebtsov, P. Angelova, S. Sokolovski, A. Abramov, E. Rafailov, "Verification of NADH content measurements by portable optical diagnostic system in living brain tissue," Proc. SPIE 10685, Biophotonics: Photonic Solutions for Better Health Care VI, 106854E (17 May 2018); doi: $10.1117 / 12.2307552$

SPIE. Event: SPIE Photonics Europe, 2018, Strasbourg, France 


\title{
Verification of NADH content measurements by portable optical diagnostic system in living brain tissue
}

\author{
E. Zherebtsov*a, P. Angelova ${ }^{\text {b }}$, S. Sokolovski ${ }^{a}$, A. Abramov ${ }^{\text {b }}$ E. Rafailov ${ }^{\mathrm{a}}$ \\ aAston Institute of Photonic Technologies, Aston University, Aston Triangle, Birmingham, B4 7ET, UK; \\ bepartment of Molecular Neuroscience, UCL Institute of Neurology, Queen Square, London, WC1N 3BG, UK.
}

\begin{abstract}
The overall aim of this study was verification of the possibility to register the change of NADH fluorescence in live tissue by a portable diagnostical laser system with fibre optical probe output and excitation by compact semiconductor UV light source. The measurements were conducted in fresh brain tissue slices of Wistar rat pups. The fluorescence measurements were conducted simultaneously at intervals of $5 \mathrm{~s}$ by the microscopic system with excitation at $360 \mathrm{~nm}$ and registering of the emitted fluorescence light at $455 \mathrm{~nm}$ and by the tested diagnostical system equipped with the fibre optical probe with excitation at $365 \mathrm{~nm}$ and registration of the fluorescence spectrum by the inbuilt spectroscopic subsystem. To modulate the mitochondrial function in the living cells, in the chamber sequentially were added $1 \mu \mathrm{M}$ FCCP and $1 \mathrm{mM} \mathrm{NaCN}$. The comparisons between the curves registered by the methods allowed us to find well agreement between the microscopic measurements and measurements by the fibre optical probe. The obtained results prove that the tested diagnostic system is capable of sensing the changes in brain metabolic activity associated with the NADH content alterations within the physiological range.
\end{abstract}

Keywords: fluorescence, NADH, optical biopsy, mitochondrial dysfunction, uncoupling agent, histotoxic hypoxia

\section{INTRODUCTION}

Cell mitochondria are leading elements in the process of live cell energy production. Pathological changes in the energy balance of the organelles lead to severe effects that can result in cell death. At present, many diseases and morbid conditions have been connected with mitochondrial dysfunction. In turn, various pernicious changes in the mitochondrial respiratory chain are recognized as one of the consequences resulting from a breach in the cell and organ function observable in different pathologies. Thus, the diagnostics of the pathologies can be implemented via measurements of the mitochondrial parameters. The most likely such changes to occur are the different accumulation of a coenzyme nicotinamide adenine dinucleotide (NADH) (the reduced form) and flavin adenine dinucleotide (FAD) (oxidised form). Starting from studies of Chance and his followers the measurements have become used in medical diagnostics. Detection of such changes by fluorescence spectroscopy is one of the promising directions for diagnosis in vivo.

Currently, a lot of research is being done to solve the challenge of the metabolism assessment in living tissues, including skin, using fluorescence spectroscopy and imaging techniques. A recent review ${ }^{1,2}$ and some other studies show that changes in the fluorescence of the NADH respiratory chain were measured in single cells, ${ }^{3,4}$ tissue slices, ${ }^{5}$ and organs. ${ }^{6}$ Despite the fact that the study of tissue fluorescence has been held for the past 50 years, relatively little research has been conducted at the organ level with verification of the source and nature of the detected signal. Meanwhile, the results of experiments on a particular organ or area of the body can be more successfully extrapolated to clinical practice in comparison to the studies at the cellular and subcellular levels.

The central problem of the fluorescence measurements on the organ level is the detection of the signal against the background of disturbing factors (such as fluorescence and absorbance of different tissue components, which is of no interest for the study). ${ }^{7}$ For the measurements in vivo, the most significant source of variability for the recording fluorescence data is the influence of variable blood absorption in the living tissue. ${ }^{8,9}$ To compensate the influence of blood content on the fluorescence measurements in vivo, a diagnostic system with fibre optical probe combining fluorescence and diffuse reflectance measurements was proposed previously (Figure 1). For the inverse solution of the blood content recovery, a set of neural networks trained by the Monte Carlo generated learning set was used. This approach has been tested in the trials of fluorescence measurements with simultaneous real-time measurements of blood content in living tissue during moderate changes in contact pressure of the optic probe..$^{10,11}$

*e.zherebtsov@aston.ac.uk; phone +44 121204 3703; www.aston.ac.uk/eas/research/groups/photonics/research-areas

Biophotonics: Photonic Solutions for Better Health Care VI, edited by Jürgen Popp,

Valery V. Tuchin, Francesco Saverio Pavone, Proc. of SPIE Vol. 10685, 106854E

(C) 2018 SPIE · CCC code: 0277-786X/18/\$18 · doi: 10.1117/12.2307552 
On the other hand, the autofluorescence measurements of the cell cofactors (NADH, NADPH, FAD) using different microscopy techniques have become routine in the assessment of mitochondrial dysfunction in many biological protocols. ${ }^{12}$ This knowledge can be transferred to build effective methods and devices for diagnostics in vivo. However, few researchers have addressed the problem of the comparison of the measuring results obtained by a microscopy method and by measurement techniques suitable for the in vivo applications. In this study, the changes in NADH fluorescence have been simultaneously measured by epifluorescence microscopy system and by the proposed diagnostic system equipped with fibre optical probe. The present paper aims to compare the results obtained by the two methods and to validate the possibility to register the changes of NADH fluorescence in live tissue by the portable diagnostic laser system with fibre optical probe output and excitation by compact semiconductor UV light source.

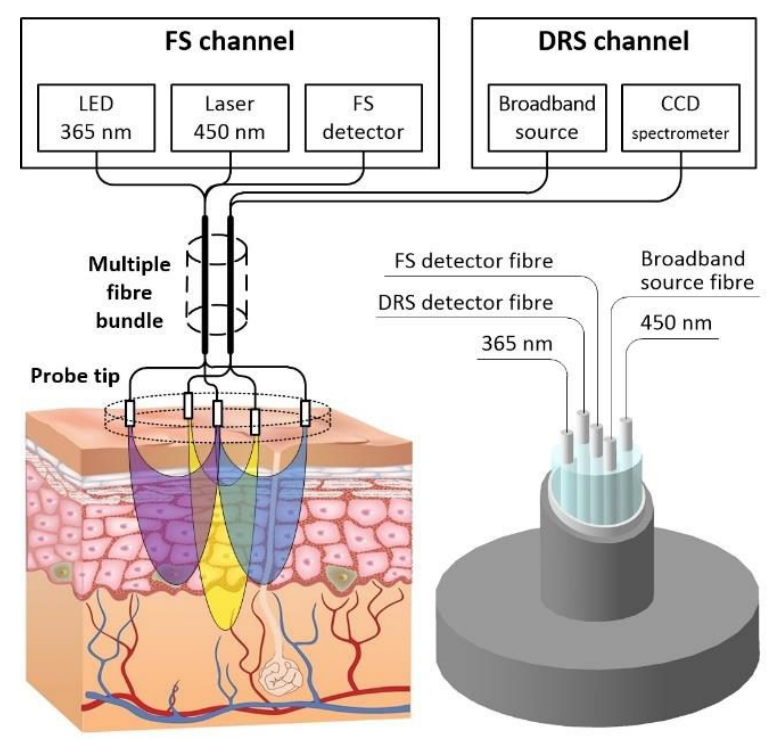

Figure 1. Measuring setup combines FS and diffuse reflectance measuring channels

\section{MATERIALS AND METHODS}

A fibre optical probe combining fluorescence ( 2 emitting and 1 collecting fibres) and diffuse reflectance measurement (1 emitting and 1 collecting fibres) approaches has been used (Figure 1). The probe was used for delivery of penetrating radiation and registration of back-reflected secondary radiation from the tissue. A $365 \mathrm{~nm}$ radiation source was used for fluorescence excitation. The probing and collecting fibres have a diameter of 400 microns. The source-detector spacing for the FS channel is $1.5 \mathrm{~mm}$ whereas the distance for the DRS channel is $2 \mathrm{~mm}$. For safety reasons, as well as to keep photobleaching of the tissue at an acceptable level, probe radiation power of the $365 \mathrm{~nm}$ excitation wavelength at the output of the fibre probe did not exceed $1.5 \mathrm{~mW}$. To assess the safety of the probe, coupled with the LED, we used documentation of the International Commission On Non-Ionizing Radiation Protection (ICNIRP) ${ }^{13}$. The calculation gives the effective skin irradiance of $1.4 \mathrm{~W} / \mathrm{m}^{2}$. From the ICNIRP recommendations, the proposed protocol of using the probe was deemed to be safe. The numerical aperture of the fibres was 0.22 .

Fluorescence imaging measurements were obtained using Nikon (Tokyo, Japan) epifluorescence inverted microscope equipped with 20x fluorite objective and a cooled CCD camera (Andor Technology Ltd, UK). Excitation light from a xenon arc lamp was selected using $10 \mathrm{~nm}$ bandpass filter housed in the computer-controlled filter wheel.

The measurements were conducted in fresh brain tissue slices of Wistar rat pups. The slices and primary neuronal cultures were isolated from the rats according to the Home Office UK regulations. For the measurements, the slices of 200 um were prepared with a microtome and immediately were put into imaging chamber with HBSS buffer of the microscope (Figure 2). Further the fluorescence measurements were conducted simultaneously at intervals of $5 \mathrm{~s}$ by the microscopic system with excitation at $360 \mathrm{~nm}$ and registering of the emitted fluorescence light at $455 \mathrm{~nm}$, and by the 
tested diagnostical system equipped with the fibre optical probe with excitation at $365 \mathrm{~nm}$ and registration of the fluorescence spectrum by the inbuilt spectroscopic subsystem. To modulate the mitochondrial function in the living cells, in the chamber sequentially were added $1 \mu \mathrm{M}$ FCCP (as uncoupling agent) and $1 \mathrm{mM} \mathrm{NaCN}$ (as Complex IV inhibitor).

The duration of every experiment was selected to sufficiently cover the transient processes in the registered signal of the fluorescence response.

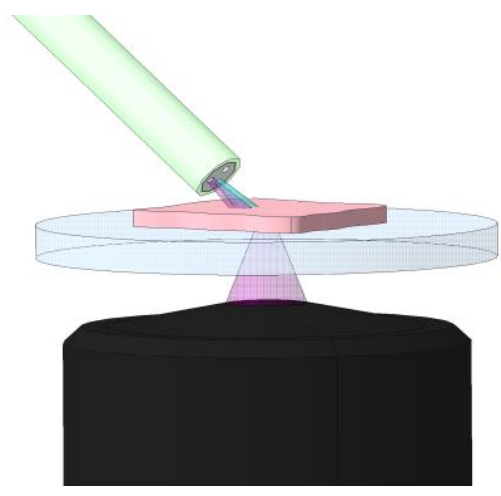

a)

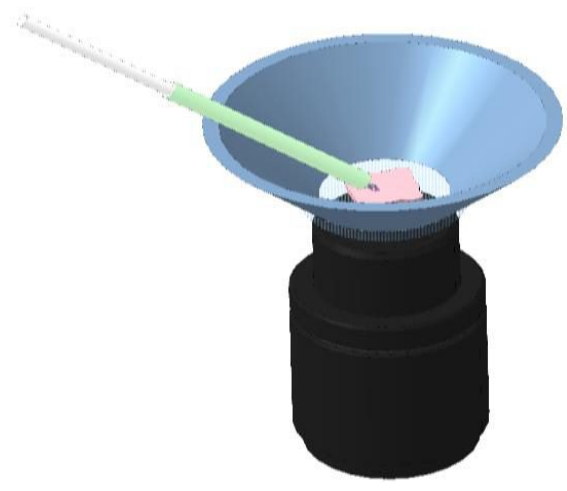

b)

Figure 2. Simultaneous measurements of NADH fluorescence in cells and living tissues by the fibre-optical diagnostical system and epifluorescence inverted microscope (a); the arrangement of the fibre-optic probe in the imaging chamber (b)

The processing of the microscopic images consisted of the selection of the cells area (15-20 regions of interest per experiment), intensity averaging and normalizing by a maximum of the obtained time-fluorescence intensity profile (Figure 3).

\section{The processing of the microscopic images}

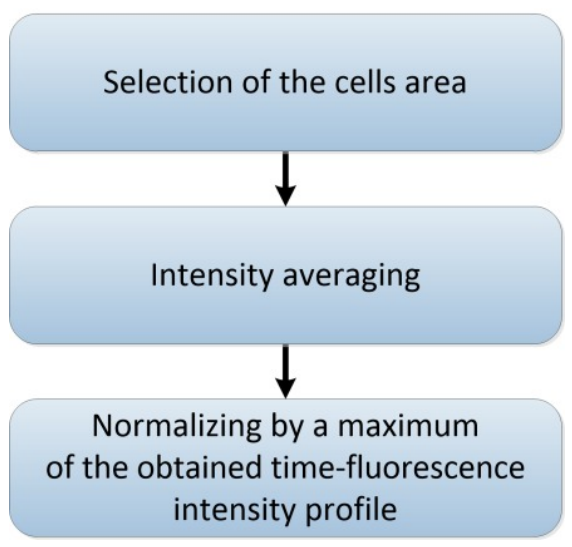

\section{The processing of the fluorescence spectra}

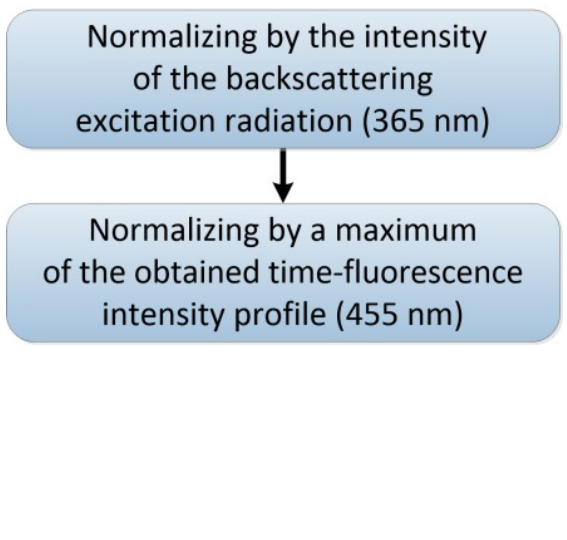

Figure 3. Schema of data processing

The obtained from the diagnostic system fluorescence spectra were normalized by the intensity of the backscattering excitation radiation and, further, the curve of the time-intensity profile for the fluorescence at $455 \mathrm{~nm}$ was scaled by normalizing by maximum intensity. 


\section{EXPERIMENTAL RESULTS AND DISCUSSION}

An example of the registered fluorescence spectrum from fresh rat brain slice is shown in Figure 4. During the simultaneous measurements, the areas of the signal averaging were significantly different (several tens of cells for microscopy and almost overall thickness of the slice for the fibre optical probe). Nevertheless, in fresh slices, the both time series obtained by the methods after the mentioned procedure of the normalising find good agreement with each other. An example of the simultaneous measurement of the fluorescence intensity in living tissue slice (about 60 minutes after sacrificing the animal) by epifluorescence microscope and diagnostic system with the fibre-optic probe is shown in Figure 5.

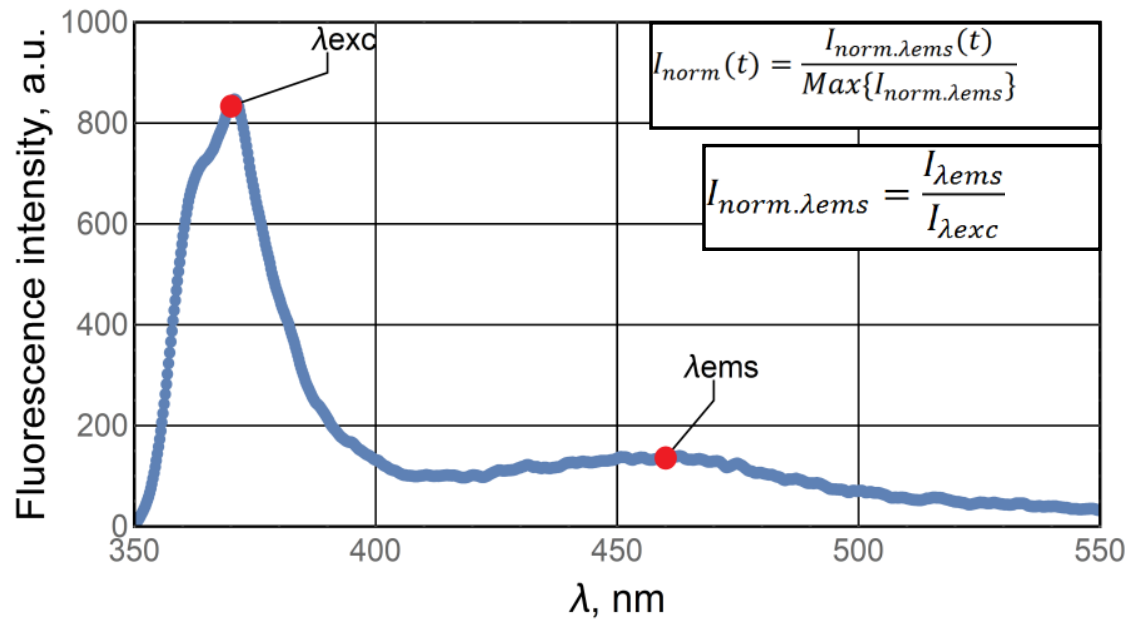

Figure 4. Example of the registered fluorescence spectrum from fresh rat brain slice

During the described procedure the FCCP induces the maximally oxidised state in cells by stimulation of maximal cellular respiration, which leads to the minimisation of the content of the mitochondrial NADH. As a result, the minimum of the registered fluorescence has been achieved. Conversely, $\mathrm{NaCN}$ provokes the maximum possible content of mitochondrial NADH due to the full inhibition of respiration. In this case, the maximum possible fluorescence intensity at the NADH emission wavelengths is observed.

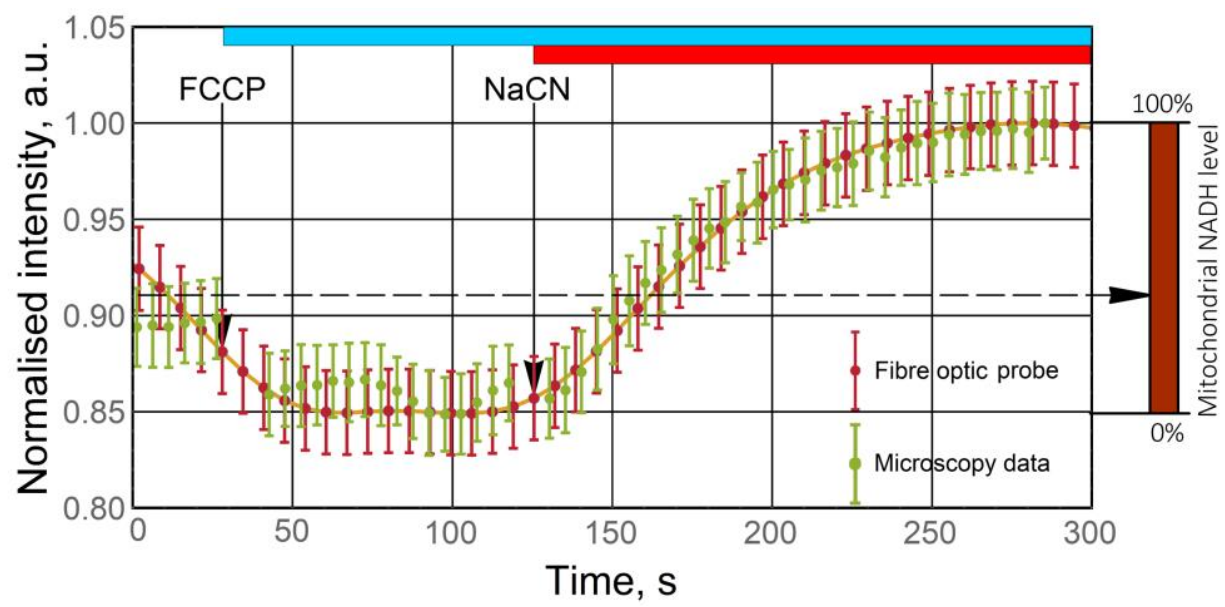

Figure 5. Simultaneous measurement (averaged) of the fluorescence intensity in living tissue slices by epifluorescence microscope and the diagnostic system with the fibre-optic probe

According to the obtained data for the measurement from Figure 5, about 15\% of the registered signal can reliably be associated with the mitochondrial NADH pool. To all appearance, the thickness of the slice was sufficient to allow the FCCP and $\mathrm{NaCN}$ to fast penetrate into the tissue, so the time delay between the registered signals for the most experiments was in the frame of the accuracy of the synchronisation between the two measurements. 
In the frame of the described procedure of simultaneous and comparative measurements, the special attention should be paid to the reliable fixation of the sample in the chamber. Whereas the averaging area for the microscopy measurements is much smaller than that for the fibre optical probe, the registration of the fluorescence intensity by the probe has demonstrated much less sensitivity to the sample movements.

\section{CONCLUSION}

The comparisons between the curves registered by the two methods allowed us to find well agreement between the microscopic measurements and measurements by the fibre optical probe. The registered signal by the probe allows quantitative parameters of the transient process during the procedure of the modulation of the mitochondrial function to be registered. The obtained results prove that the tested diagnostic system is capable of sensing the changes in brain metabolic activity associated with the NADH content alterations within the physiological range. The system for fluorescence diagnostics with blood influence compensation supplemented by the channel for the fluorescence lifetime measurements is supposed to be applied in vivo diagnostics with potential application in cancer diagnostics, organ transplants, drug discovery and cosmetics.

\section{ACKNOWLEDGEMENTS}

The reported study has received funding from the European Union's Horizon 2020 research and innovation programme under the Marie Skłodowska-Curie grant agreement No. 703145.

\section{REFERENCES}

[1] A. Mayevsky and B. Chance, "Oxidation-reduction states of NADH in vivo: from animals to clinical use," Mitochondrion 7(5), 330-339 (2007).

[2] A. Mayevsky and G. G. Rogatsky, "Mitochondrial function in vivo evaluated by NADH fluorescence: from animal models to human studies.," Am. J. Physiol. Cell Physiol. 292(2), 615-40 (2007).

[3] F. Bartolome and A. Y. Abramov, "Measurement of mitochondrial NADH and FAD autofluorescence in live cells," Methods Mol Biol 1264, 263-270 (2015).

[4] K. M. Holmstrom et al., "Nrf2 impacts cellular bioenergetics by controlling substrate availability for mitochondrial respiration," Biol. Open 2(8), 761-770 (2013).

[5] K. A. Foster, C. J. Beaver, and D. A. Turner, "Interaction between tissue oxygen tension and NADH imaging during synaptic stimulation and hypoxia in rat hippocampal slices.," Neuroscience 132(3), 645-657 (2005).

[6] G. Papayan, N. Petrishchev, and M. Galagudza, "Autofluorescence spectroscopy for NADH and flavoproteins redox state monitoring in the isolated rat heart subjected to ischemia-reperfusion," Photodiagnosis Photodyn Ther 11(3), 400-408 (2014).

[7] V. V. Dremin et al., "Multimodal optical measurement for study of lower limb tissue viability in patients with diabetes mellitus," J. Biomed. Opt. 22(8), 1-10 (2017).

[8] L. Lim et al., "Probe pressure effects on human skin diffuse reflectance and fluorescence spectroscopy measurements," J. Biomed. Opt. 16(1), 011012 (2011).

[9] V. V. Dremin et al., "The blood perfusion and NADH/FAD content combined analysis in patients with diabetes foot," Proceedings of SPIE 9698, 1-10 (2016).

[10] E. Zherebtsov et al., "Fibre-optic probe for fluorescence diagnostics with blood influence compensation," Proceedings of SPIE 10493, 1-6 (2018).

[11] E. A. Zherebtsov et al., "The influence of local pressure on evaluation parameters of skin blood perfusion and fluorescence," Proceedings of SPIE 10336, 1-10 (2017).

[12] P. R. Angelova and A. Y. Abramov, "Role of mitochondrial ROS in the brain: from physiology to neurodegeneration," FEBS Lett. 592(5), 692-702 (2018).

[13] "Guidelines on limits of exposure to ultraviolet radiation of wavelengths between $180 \mathrm{~nm}$ and $400 \mathrm{~nm}$ (incoherent optical radiation)," Health Phys. 87(2), 171-186 (2004). 